\title{
АНАЛИЗ УРОВНЯ УСТОЙЧИВОГО ПОТРЕБЛЕНИЯ В КОНТЕКСТЕ ЦИФРОВОЙ ИНФОРМАЦИОННОЙ СРЕДЫ
}

\author{
(c) 2020 Конников Евгений Александрович \\ кандидат экономических наук, доцент, Высшая инженерно-экономическая школа \\ Санкт-Петербургский политехнический университет Петра Великого (СПбПУ), \\ Россия, Санкт-Петербург \\ E-mail: konnikov.evgeniy@gmail.com \\ (c) 2020 Терентьева Дарья Андреевна \\ магистрант \\ Санкт-Петербургский государственный университет (СПбГУ), Россия, Санкт-Петербург \\ E-mail:dulybina@mail.ru

\section{(c) 2020 Конникова Ольга Анатольевна} \\ кандидат экономических наук, доцент, кафедра маркетинга \\ Санкт-Петербургский государственный экономический университет (СПбГЭУ), \\ Россия, Санкт-Петербург \\ E-mail: olga.a.konnikova@gmail.com
}

Цель проводимого исследования заключается в построении инструмента сравнительной оценки уровня устойчивого потребления. Авторами было установлено, что свойства устойчивого потребления могут проявляться в рамках 2 параллельных сред: объективной среде, являющейся совокупностью вещественных объектов и их трансформации; и информационной среде, являющейся совокупностью информационных объектов и их трансформации. Элементарные информационные единицы и их совокупности были в свою очередь разделены на две базовые компоненты: содержательнотематическая компонента и тональная компонента. Авторами был разработан укрупненный алгоритм оценки относительного присутствия определенной содержательно-тематической компоненты в информационном фоне исследуемого субъекта, апробированный при оценке уровня устойчивого потребления участников 3 студенческих сообществ в социальной сети vk.com, посвященных вопросам устойчивого потребления. Сформированный инструмент позволяет оценить уровень устойчивого потребления, или степень устойчивости потребления, для целей сравнительного анализа информационных субъектов или их совокупностей. На основе данного сравнительного анализа может быть определен целевой сегмент управленческого или маркетингового воздействия, а также идентифицирована совокупность потенциальных потребителей продуктов, конкурентным преимуществом которых является соответствие концепции устойчивого потребления.

Ключевые слова: устойчивое потребление, оценка уровня устойчивого потребления, информационная среда, содержательно-тематическая компонента, тональная компонента.

В современном мире потребительская культура [1] развивается с каждым годом благодаря доступности различных материальных благ. На данный момент наблюдается тенденция, которая заключается в приобретении как можно большего количества товаров, вне зависимости от степени их необходимости для индивидуума [2]. Так, люди удовлетворяют свою потребительскую потребность, акцентируя внимание на процессе получения различной продукции, а не ее использования. Одним из ключевых факторов, влияющих на данную ситуацию, является развитие рекламного сектора в аспектах психологического воздействия на человека [3] и технологического прогресса в визуальной культуре. Кроме того, капиталистическая система многих стран способствует распространению идеи об эквивалентности таких понятий как «успех» и «прибыль», а наличие средств может быть отражено через приобретение как можно большего количества благ. В совокупности это приводит к повсеместному распространению соответствующего паттерна потребительского поведения. Последствия, с которыми сталкивается мировое 
сообщество из-за данного подхода к потреблению, заключаются в отклонении от принципов устойчивого развития [4], в частности в нанесении ущерба окружающей среде.

На текущий момент вопросы экологии являются одними из первостепенных на глобальном уровне. Это связано с ухудшением экологической обстановки и такими проблемами как загрязнение пластиком мирового океана [5], изменение климата [6] и многие другие. Инфраструктура и технологии развиваются, однако эти изменения несут в себе угрозу для окружающей среды, а чрезмерное потребление лишь усугубляет сложившуюся ситуацию. Таким образом, необходимо сокращать оказываемое человечеством воздействие на окружающую среду, одной из возможностей подобного сокращения является переход к устойчивому потреблению.

Устойчивое потребление представляет собой концепцию, при которой индивидуум приобретает и использует лишь необходимое и достаточное количество продукции, сокращая таким образом отходы, а также делает выбор в пользу наиболее экологически дружественных товаров и услуг [7]. Благодаря тому, что на устойчивые продукты спрос увеличивается, производители также вынуждены переходить к устойчивому производству, тем самым снижая воздействие на окружающую среду. Если данная концепция будет использоваться повсеместно на различных уровнях, начиная с индивидуального и заканчивая государственным, это значительно повлияет на экологическую обстановку в мире в положительном ключе.

Устойчивое потребление на данный момент все больше распространяется в странах, у которых первым приоритетом стоит следование концепции устойчивого развития, например, в Швеции. Для того, чтобы предпринимать какие-либо действия по увеличению уровня устойчивого потребления, необходимо определить его первоначальное значение на индивидуальном уровне.

Представленное авторами исследование проводится методом анализа информационного потока в тематических сообществах в социальных сетях. Поскольку концепция устойчивого потребления основана на принципе осознанности, то обсуждение данной темы в общедоступном пространстве также является проявлением осознанности и может быть интерпретирован как уровень устойчивого потребления.
Проблема, которую авторы решают в ходе проведения текущего исследования, заключается в определении уровня устойчивого потребления. Данная проблема действительно важна и актуальна, потому что потребление благ является неотъемлемой частью жизни человека, которую можно изменить так, чтобы воздействие на окружающую среду снизилось. А вопросы экологии становятся все более важными с каждым годом в связи с ухудшением экологической обстановки в мире.

Цель исследования заключается в том, чтобы построить инструмент сравнительной оценить уровня устойчивого потребления и предложить мероприятия по повышению данного показателя.

Устойчивое потребление представляет собой использование услуг и сопутствующих товаров, которые отвечают основным потребностям и улучшают качество жизни при уменьшении использования природных ресурсов и токсичных материалов, а также отходов и загрязнения на протяжении всей жизни услуг или продукта так, чтобы не подвергать опасности нужды будущих поколений. Концепция устойчивого потребления была сформулирована на симпозиуме в Осло в 1994 году [8].

Данная тенденция получила широкое развитие в современном мире, поскольку она способствует достижению целей устойчивого развития. Цели в области устойчивого развития на период до 2030 года (ЦУР) представляют собой амбициозный пакет из 17 глобальных целей на период до 2030 года, устанавливающие новую парадигму экономического и социального развития, включая множество целей, связанных с решением экологических проблем [9].

Идея «устойчивости» как направления развития была впервые сформулирована в 1987 году в отчете «Наше общее будущее» специальной комиссии по окружающей среде и развитию под покровительством ООН [10]. Тогда было определено, что устойчивое развитие - это такое развитие, которое позволяет удовлетворять потребности нынешнего поколения без ущерба для возможности будущих поколений удовлетворять свои собственные потребности. На конференции ООН по окружающей среде и развитию в Рио-де-Жанейро в 1992 году были приняты положения устойчивого развития и сформулированы действия для его осуществления [11]. 
Устойчивое развитие представляет собой качественную концепцию, которая охватывает экономическую и социальную сферы, а также рассматривает окружающую среду. Таким образом, данный феномен включает в себя политические аспекты, нацеленные на улучшение социальной обстановки и достижение «равных возможностей» для всех граждан, а также природоохранные и экономические меры, направленные на эффективное использование ресурсов и сокращения вреда окружающей среде.

Устойчивое потребление является одним из аспектов устойчивого развития, и именно этот аспект на текущий момент рассматривается в наибольшей степени. Это связано с развитием культуры потребительского поведения и увеличением потребления, так как эти взаимосвязанные явления приводят к возрастанию ущерба окружающей среде. Неустойчивое потребление в основном характеризует развитые страны, так как именно в них значительное приобретение различных благ удовлетворяет потребительские потребности людей, а не использование товаров [12]. Однако в развивающихся странах также есть тенденция увеличения «неустойчивости» потребления, в частности, в домашнем секторе. Исследования показывают, что потребление в секторе домашних хозяйств Европейского союза прямо или косвенно - через использование услуг и продуктов - обуславливает приблизительно 70\% воздействия на окружающую среду.

Устойчивое потребление может рассматриваться на различных уровнях, и равномерное распределение ресурсов, в соответствии с определением устойчивого развития, может осуществляться между государствами и регионами, а также внутри государств.

В контексте принципов устойчивого потребления рассматриваются следующие понятия:

- Достаточность

- Экологически дружественное потребление

- Снижение использования природных ресурсов

- Сокращение отходов

Достаточность подразумевает такое потребление материальных благ, при котором достигается лучшее качество жизни и удовлетворяются текущие потребности, однако отсутствует чрезмерное потребление товаров и услуг.

Суть экологически дружественного потребления заключается в предпочтении тех товаров и услуг, при производстве и предоставлении которых компании оказывают наименьший вред окружающей среде и соответствуют всем экологическим стандартам [13].

Снижение использования природных ресурсов также соответствует определению «устойчивости», что связано с необходимостью обеспечения последующих поколений достаточным количеством ресурсов для удовлетворения потребностей. Таким образом, данный аспект нацелен на сохранение исчерпаемых природных ресурсов и разработку альтернатив данных ресурсов для их дальнейшего безопасного потребления.

Сокращение отходов и сведение их к нулю, то есть осуществление переработки - основа циркулярной экономики [14]. Которая, в свою очередь, стремится максимально эффективно использовать все ресурсы и не загрязнять окружающую среду опасными отходами производства и потребления.

Таким образом, каждый из вышеперечисленных аспектов демонстрирует проявление одной из основных целей всех «устойчивых» инициатив, а именно снижение воздействия на окружающую среду. Примерами такого воздействия, возникшего в результате неустойчивого потребительского поведения, являются изменение климата, эвтрофикация, сокращение стратосферного слоя озона [15].

Рассматривая устойчивое потребление в контексте повседневной жизни человека, можно выделить следующие сферы наиболее высокого влияния на окружающую среду:

1. Потребление пищевых продуктов и напитков

2. Потребление в жилом помещении

3. Транспортная мобильность

Данные категории расположены в соответствии с величиной степени влияния на окружающую среду в вопросе устойчивого потребления.

Потребление пищевых продуктов и напитков

Устойчивое (сбалансированное) потребление пищи может быть определено как равный для всех поколений доступ к потреблению пищи, необходимой для поддержания активной и здоровой жизни; при этом способы добывания и потребления пищевых продуктов должны отвечать требованиям устойчивости в экономическом, социальном отношении и в аспекте охраны окружающей среды [16]. 
В силу необходимости данных товаров для человека, эта сфера является неотъемлемой частью нашей жизни, и неустойчивое потребление в рассматриваемой категории приводит к значительному воздействию на окружающую среду. Так, одна треть негативного воздействия на окружающую среду в Европе связана именно с потреблением пищевых продуктов и напитков.

При рассмотрении устойчивого потребления пищи используются следующие критерии:

- Экономический

- Социальный

- Природоохранный

С экономической точки зрения рассматриваются вопросы торговли и различных экономических показателей, таких как прибыль; социальный критерий предусматривает аспект здоровья человека, безопасности потребляемой пищи, качества жизни; а природоохранный отвечает за использование природных ресурсов, загрязнение окружающей среды. Все три компонента устойчивого потребления и производства пищи взаимосвязаны, оказывают влияние друг на друга и составляют интегрированное целое.

Устойчивое потребление пищи нельзя рассматривать в отрыве от производства, так как состав потребляемых продуктов имеет огромное значение. Таким образом, на «устойчивость» потребления пищи влияет процесс производства и переработки, распределения и продажи, а в некоторых ситуациях транспортировки.

Одним из предлагаемых способов создания модели более устойчивого потребления пищи является предложение сокращать производство пищевых продуктов, требующих невозобновляемых природных ресурсов, и заменить одни продукты другими. Другие направления - поощрение потребления сезонных продуктов и продуктов местного производства; постепенное изменение укоренившихся привычек, связанных с питанием; потребление меньшего, но адекватного для удовлетворения потребностей организма, количества пищи; поиск новых парадигм, побуждающих к устойчивому потреблению пищевых продуктов.

\section{Потребление в жилом помещении}

В данной категории потребления основной акцент делается на следующих аспектах потребления в повседневной жизни:

- Вода

- Электроэнергия

- Тепловая энергия
- Образование бытовых отходов

В настоящее время запасы воды как неисчерпаемого природного ресурса сокращаются в связи с перерасходованием и загрязнением, в то время как потребность в ней увеличивается пропорционально росту населения и развитию экономики. Более других источников запасов пресной воды подвержены опасности подземные воды. Это связано с особенностями процесса их загрязнения и дальнейшими сложностями с определением характера ущерба подземным водам.

Ввиду нахождения данного ресурса глубоко под землей, существуют значительные трудности с восстановлением и очищением вод. А поскольку подземные воды используются наиболее часто в сопоставлении с другими природными ресурсами, неустойчивое потребление воды может привести к кризису, связанному с ее запасами.

В контексте потребления в жилых помещениях устойчивое потребление воды может быть отражено в экономном использовании данного ресурса и наименьшем загрязнении по мере возможностей.

Экономия в энергетическом аспекте потребления в жилых помещениях обусловлена способом производства электрической и тепловой энергии. Рост энергопотребления оказывает прямое вредное воздействие на окружающую среду и изменение климата: выброс загрязняющих среду веществ причиняет вред людям, экосистемам, расходуются природные ресурсы [17]. Изменение климата вызвано повышенной концентрацией в атмосфере газов, создающих парниковый эффект (фторосодержащие газы, оксиды азота, метан, углекислый газ). Увеличивающиеся выбросы $\mathrm{CO}_{2}$ и других газов, провоцирующих парниковый эффект, большей частью связаны со сжиганием ископаемых видов топлива [18]. Главные отрасли использования этого вида топлива - энергетика и транспорт.

Таким образом, при сокращении энергопотребления повышается его «устойчивость».

Бытовые отходы - это любые материалы и вещи, которые образуются в быту человека ежедневно, вне зависимости места проживания (многоквартирный дом, частный дом и т.д.). Они могут быть представлены пищевыми отходами, бытовой химией, упаковочными материалами, отходами электрического и электронного оборудования, мебелью. 
Для повышения «устойчивости» в данном аспекте необходимо утилизировать отходы правильно, а именно использовать систему раздельного сбора отходов [19], а при ее отсутствии сдавать отдельные категории использованных товаров на переработку.

\section{Транспортная мобильность}

Урбанизация и постоянное экономическое развитие отражаются и на таком секторе как транспорт. Повышение использования личного транспорта в противовес общественному значительно отражается на окружающей среде. Существуют следующие виды воздействия:

- Выбросы СО2 и вклад в изменение климата

- Загрязнение воздуха

- Шум

- Сокращение биоразнообразия

В качестве примера устойчивого потребления в данном вопросе можно привести полный отказ от личного автомобиля в пользу общественного транспорта или экологически безопасного транспортного средства, например, велосипеда. Также переход на биологическое топливо может снизить негативное воздействие продуктов сгорания на окружающую среду [20].

Таким образом, в современном мире с доминирующей культурой потребления необходимо придерживаться принципов устойчивости в данном вопросе, чтобы предотвратить нанесение значительного ущерба окружающей среде [21]. Каждая из рассмотренных категорий повседневной жизни человека влияет на глобальные экологические проблемы современности, поэтому внедрение понятия «устойчивости» и рассмотрение уровня осознанности населения в данном вопросе являются первостепенными задачами в контексте устойчивого развития [13, 25].

В соответствии с проведенным теоретическим исследованием можно заключить, что процесс устойчивого потребления может быть охарактеризован посредством множества характеристик, которые могут быть измерены как в количественной шкале, так и в порядковой и номинальной. K количественно измеряемым характеристикам, отражающим степень устойчивости потребления, можно отнести объем средств, направляемых на благотворительность, доля эко товаров в потребительской корзине оцениваемого субъекта и многое другое. Однако, наиболее значительная часть характеристик, отражающих степень устойчивости потребления, являются качественными, и могут быть оценены исключительно экспертно или посредством иных эвристических методов анализа. Также стоит отметить, что на данный момент собираемая в области устойчивого потребления статистическая информация носит исключительно локальный характер, является следствием отдельных социологических и маркетинговых исследования, и в большинстве случаев не может быть универсализирована и экстраполирована для целей формирования методологических инструментов анализа и управления. Рассмотренные ранее аналитический подходы и инструменты также не могут быть универсализированы для целей формирования инструментария методологического уровня. Следовательно, отправной точкой процесса формирования инструментария оценки степени устойчивости потребления, или уровня устойчивого потребления, является исключительно сформированный общетеоретический базис.

Как было установлено ранее, под устойчивым потреблением следует понимать процесс использования ТРУ, которые отвечают основным потребностям и улучшают качество жизни при уменьшении использования природных ресурсов и токсичных материалов, а также отходов и загрязнения на протяжении всей жизни услуг или продукта. Так, можно заключить, что процесс потребления дифференцируется на 2 базовые компоненты:

1. Приращение личного блага субъекта потребления. Данное приращение является естественным свойством любого процесса потребления.

2. Приращение общественного блага, выраженное в уменьшении использования природных ресурсов и токсичных материалов, а также отходов и загрязнения.

Данные компоненты, при условии их максимизации, характеризуются обратной функциональной зависимостью. Следовательно, устойчивое потребление подразумевает формирование баланса количественных интерпретаций данных комплексных компонент. Как уже отмечалось ранее, проявление каждой из выделенных компонент может быть выражено посредством множества качественных и количественных переменных. Однако, сами свойства устойчивого потребления могут проявляться в рамках 2 параллельных сред:

1. Объективной среде, являющейся сово- 
купностью вещественных объектов и их трансформации.

2. Информационной среде, являющейся совокупностью информационных объектов и их трансформации.

Большая часть проводимых на данный момент исследований в области устойчивого потребления нацелены на анализ результатов трансформации объективного мира, таких как снижение объема наносимого экологического и социального ущерба в процессе потребления, приращение запасов природных ресурсов и многое другое. Анализ данных результирующих параметров, как и в целом анализ трансформации характеристик объективного мира, сопряжен с множеством естественных (физических) ограничений. При этом информационная среда является значительно более доступной для анализа. Данный факт является следствием развития информационного общества и цифровизации основных экономических и социальных процессов. Массовое развитие интернеттехнологий спровоцировало цифровизацию социальной коммуникации, следствием чего стало повсеместное проникновение интернет СМИ и социальных сетей. Информационная среда не является иерархически управляемой и централизованной, что указывает на потенциальную достоверность генерируемых и транслируемых в рамках нее информационных потоков. При этом трансформации объективного мира неизбежно отражаются в информационной среде. В рамках данного исследования выдвигается гипотеза, согласно которой результаты устойчивого потребления отражаются в объектах информационной среды, и их трансформации. Следовательно, квантификация уровня устойчивого потребления может быть реализована посредством анализа информационной среды.

Объектами информационной среды являются элементарные информационные единицы, закодированные в текстовой или аудиовизуальной форме и формирующие информационных фон, центрирующийся относительно элементов объективного мира и комплексно описывающий процесс их трансформации и взаимодействия. Данные элементарные информационные единицы генерируется субъектами информационной среды в рамках специализированных источников концентрации информации (СМИ, социальных сетей, тематических и профессиональных сообществ и т.д.). Уровень развития информационной среды во многом может характеризоваться сложностью данного информационного фона. На данный момент, в рамках определенной части источников концентрации информации значимостью обладает не только первичная информация, но и метаинформация, дифференцированная на множество уровней, и содержащая такие компоненты, как деловая репутация источника генерации, деловая репутация подавляющей части потребителей, корреляция с общеинформационным контекстом и многое иное.

Дифференцировать как элементарные информационные единицы, так и их совокупности можно на 2 базовые компоненты:

1. Содержательная-тематическая компонента, отражающая многомерную лексическую специфику элементарной информационной единицы или их совокупности.

2. Тональная компонента, отражающая свойства эмоциональной специфики элементарной информационной единицы или их совокупности.

Следует предположить, что идентификация, структурирование, квантификация и сравнительный анализ содержательно-тематической компоненты элементарной информационной единицы или их совокупности позволит охарактеризовать текущие свойства и процесс их трансформации, применительно не только комплексным элементам информационной среды, но и комплексным элементам объективного мира, одним из которых является уровень устойчивого потребления, или степень устойчивости потребления [22]. Таким образом, можно выдвинуть гипотезу, согласно которой для целей сравнительно анализа уровня устойчивого потребления базисом может выступать совокупность элементарных информационных единиц, отобранных в соответствии со спецификой объекта исследования и агрегированных в единый массив.

Для целей исследования определенной содержательно-тематической компоненты информационной среды могут быть использованы инструменты компьютерной лингвистики, в частности, алгоритмы токенизации. По результатам процесса токенизации формируется массив лексем, который может выступать отражением определенной содержательно-тематической компоненты информационной среды. Таким образом, выдвинутая ранее гипотеза может 
быть математически интерпретирована в форме коэффициента относительного присутствия содержательно-тематической компоненты устойчивого потребления в информационном фоне исследуемого субъекта (или совокупности субъектов), который может быть универсально представлен в информационной среде социального сообщества. На рисунке 1 представлен укрупненный алгоритм оценки относительного присутствия определенной содержательнотематической компоненты в информационном фоне исследуемого субъекта.

Приведенный алгоритм предполагает параллельное формирование совокупности токенов как содержательно-тематической компоненты информационной среды, так и информационной среды социального сообщества, по результатам чего формируется 2 массива токенов. Квантификация присутствия содержательнотематической компоненты в информационной среде социального сообщества предполагает расчет определённого ранее сравнительного коэффициента, который в изолированной форме не обладает аналитической ценностью. Аналитическую ценность данный коэффициент приобретает в условиях сравнения нескольких объектов (социальных сообществ).

Для целей инструментального уточнения данного алгоритма в рамках данного исследования проводится оценка уровня устойчивого потребления нескольких социальных сообществ. Для целей сравнительного анализа были определены 3 студенческих сообщества в социальной сети vk.com, посвященных вопросам устойчивого потребления:

1. «Зелёная Вышка в Санкт-Петербурге» (https://vk.com/spbgreenhse), агрегирующее студентов Высшей Школы Экономики СанктПетербурга.

2. «ITMO.GREEN» (https://vk.com/itmo_green), агрегирующее студентов Университета Информационных Технологий, Механики и Оптики.

3. «ReGreen» (https://vk.com/regreen_polytech), агрегирующее студентов Санкт-Петербургского Политехнического Университета Петра Великого.

Выборка обусловлена тем, что данные сообщества являются тематически единообразными, а следовательно, сопоставимыми, они посвящены одному из базовых аспектов устойчивого потребления, а также по той причине, что студенческая часть общества является наиболее активной в информационной среде [23]. Для целей формирования совокупности токенов содержательно-тематической компоненты информационной среды было выбран сообщество

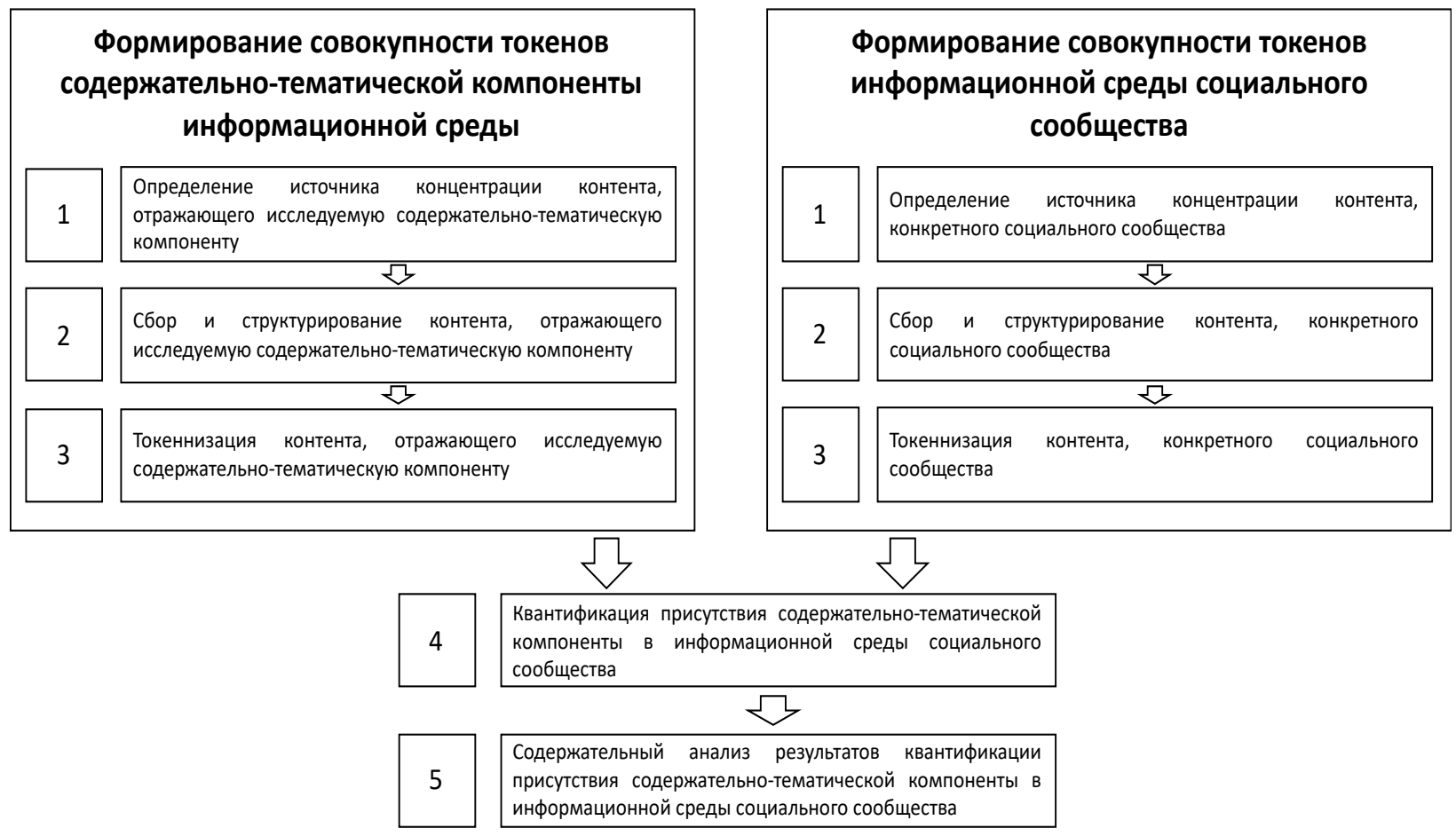

Рисунок 1. Укрупненный алгоритм оценки относительного присутствия определенной содержательнотематической компоненты в информационном фоне исследуемого субъекта 
в социальной сети vk.com «Экология» (https:// vk.com/ecoonf). Данный выбор обусловлен тем, что данный паблик является наиболее активным и востребованным в русском сегменте интернета, посвященном проблемам экологии и защиты окружающей среды (его совокупная аудитория составляет около 19 тысяч человек, и в нем каждый день размещается минимум 3 тематических поста).

Для целей автоматизации процесса формирования совокупности токенов содержательнотематической компоненты информационной среды и информационной среды социального сообщества использовался язык программирования Python 3. Алгоритм, автоматизирующий первичные этапы анализа приведен на рисунке $2[24]$.

Как можно заключить в соответствии с приведенным алгоритмом, первичная и вторичная токенизация единообразна для обоих исследуемых элементов, в то время как третий этап алгоритма - формирование аналитического Dataframe - реализуется исключитель- но для источника, отражающего исследуемую содержательно-тематическую компоненту.

Для целей квантификация присутствия содержательно-тематической компоненты в информационной среде социального сообщества разработан универсальный коэффициент - Коэффициент Содержательно-тематической Компоненты (КСС). Данный коэффициент отражает сравнительный уровень присутствия содержательно-тематической компоненты информационной среды в единице (или совокупности единиц) информационной среды социального сообщества, и определяется следующим образом:

$$
\text { KCC }=\sum_{1}^{n} T_{n} * y_{n}
$$

Где:

1. $\mathrm{T}_{\mathrm{n}}$ - сумма упоминаний токена $\mathrm{n}$ в единице (или совокупности единиц) информационной среды социального сообщества.

2. $\mathrm{y}_{\mathrm{n}}$ - сглаженная теоретическая частота (коэффициент значимости) токена $\mathrm{n}$.

1. Первичная токеннизация

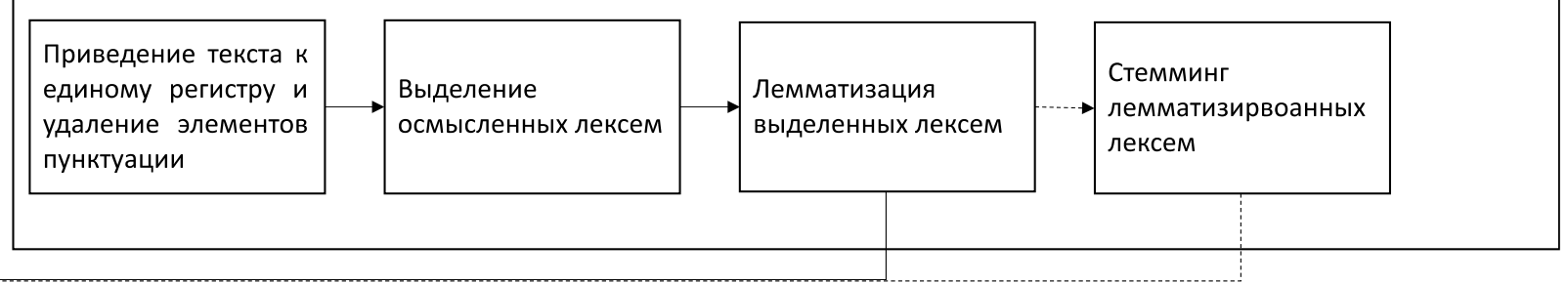

2. Вторичная токеннизация

$\rightarrow \begin{aligned} & \text { Частотная } \\
& \begin{array}{l}\text { квантификация } \\
\text { сформированного } \\
\text { массива лексем }\end{array}\end{aligned} \rightarrow$\begin{tabular}{l}
$\begin{array}{l}\text { Формальная фильтрация } \\
\text { массива лексем (удаление } \\
\text { от 10\% до 25\% наименее } \\
\text { часто } \\
\text { лексем) }\end{array}$ \\
\hline
\end{tabular}

Экспертная оценка полноты
сформированного массива
лексем. В случае положительной
оценки - агрегирование единого
массива токенов

Экспертная фильтрация массива лексем (удаление лексем, обладающих низкой содержательной значимостью)

3. Формирование аналитического Dataframe, отражающего исследуемую содержательно-тематическую компоненту

\begin{tabular}{|c|c|c|}
\hline $\begin{array}{lc}\text { Сортировка } & \text { сформированного } \\
\text { массива } & \text { токенов }\end{array}$ & $\begin{array}{l}\text { Аппроксимация } \\
\text { посредствам } \text { экстоты } \\
\text { функции: } y=a * e^{b * x} \text {, где у - }\end{array}$ & $\begin{array}{l}\text { Расчет коэффициентов значимости } \\
\text { токенов } и \text { и формирование } \\
\text { двумерного DataFrame, содержащего }\end{array}$ \\
\hline $\begin{array}{l}\text { соответствии с увеличением } \\
\text { частоты }\end{array}$ & $\begin{array}{l}\text { сглаженная теоретическая частота } \\
\text { (коэффициент значимости токена), } \\
\text { x- порядковый номер токена }\end{array}$ & $\begin{array}{l}\text { токены, упорядоченные } \\
\text { соответствии с с у увеличением } \\
\text { коэффициентов значимости }\end{array}$ \\
\hline
\end{tabular}

Рисунок 2. Алгоритм формирования совокупности токенов содержательно-тематической компоненты информационной среды и информационной среды социального сообщества 
В рамках данного исследования было идентифицировано 279 токенов, функция сглаживания для которых приобрела следующую форму:

$$
y_{n}=39.386 * e^{0.0119 * x_{n}}
$$

Где:

1. $\mathrm{x}_{\mathrm{n}}-$ порядковый номер токена $\mathrm{n}$ (токены упорядочены по увеличению частоты упоминания в информационной среде сообщества «Экология»).

Коэффициент детерминации данной модели 0.941 , что указывает на высокое качество модели, F-критерий Фишера и p-значение при $\mathrm{x}_{\mathrm{n}}$ указывают на достоверность модели и высокую значимость экзогенной переменной.

По результатам квантификации был сформирован аналитический dataframe, сформированный из оценки каждого отдельно поста. Для целей сравнительного анализа в рамках специфики данного исследования эффективнее всего использовать однофакторный дисперсионный анализ. В таблице 1 приведены результаты однофакторного дисперсионного анализа.

В соответствии с полученными результатами, можно заключить, что анализируемые сообщества значимо различны в соответствии с коэффициентом содержательной тематической компоненты «Устойчивое потребление», на что в первую очередь указывает величина р-значения 0,0032. Следовательно, несмотря на тематическое единообразие, уровень устойчивости потребления проявляется в каждом из анализируемых студенческих сообществ по-разному. Сравнение средних и дисперсий для каждого из элементов выборки приведено на рисунке 3.

В соответствии с представленными на графике результатами можно заключить, что комплексные свойства устойчивого потребления в большей степени проявляются в сообществе студентов Санкт-Петербургского Политехнического Университета Петра Великого - «ReGreen». Однако, для данного сообщества также свойственна наибольшая дисперсия, что указывает на неоднородность представленного контента с точки зрения наличия в нем содержательнотематической компоненты устойчивого потребления. Наименьшее значение исследуемого коэффициента наблюдается у студентов Высшей Школы Экономики Санкт-Петербурга - «Зелёная Вышка в Санкт-Петербурге». При этом дисперсия также сравнительно высока. Наименьший уровень дисперсии наблюдается в сообществе

Таблица 1. Дисперсионный анализ

\begin{tabular}{|l|c|c|c|c|c|c|}
\hline \multicolumn{1}{|c|}{ Источник вариации } & SS & $d f$ & MS & $F$ & Р-Значение & $\begin{array}{c}F \\
\text { критическое }\end{array}$ \\
\hline Между группами & 32968577,78 & 2 & 16484288,89 & 5,76 & 0,0032 & 3,004 \\
\hline Внутри групп & 3118333032 & 1090 & 2860855,992 & & & \\
\hline Итого & 3151301609 & 1092 & & & & \\
\hline
\end{tabular}

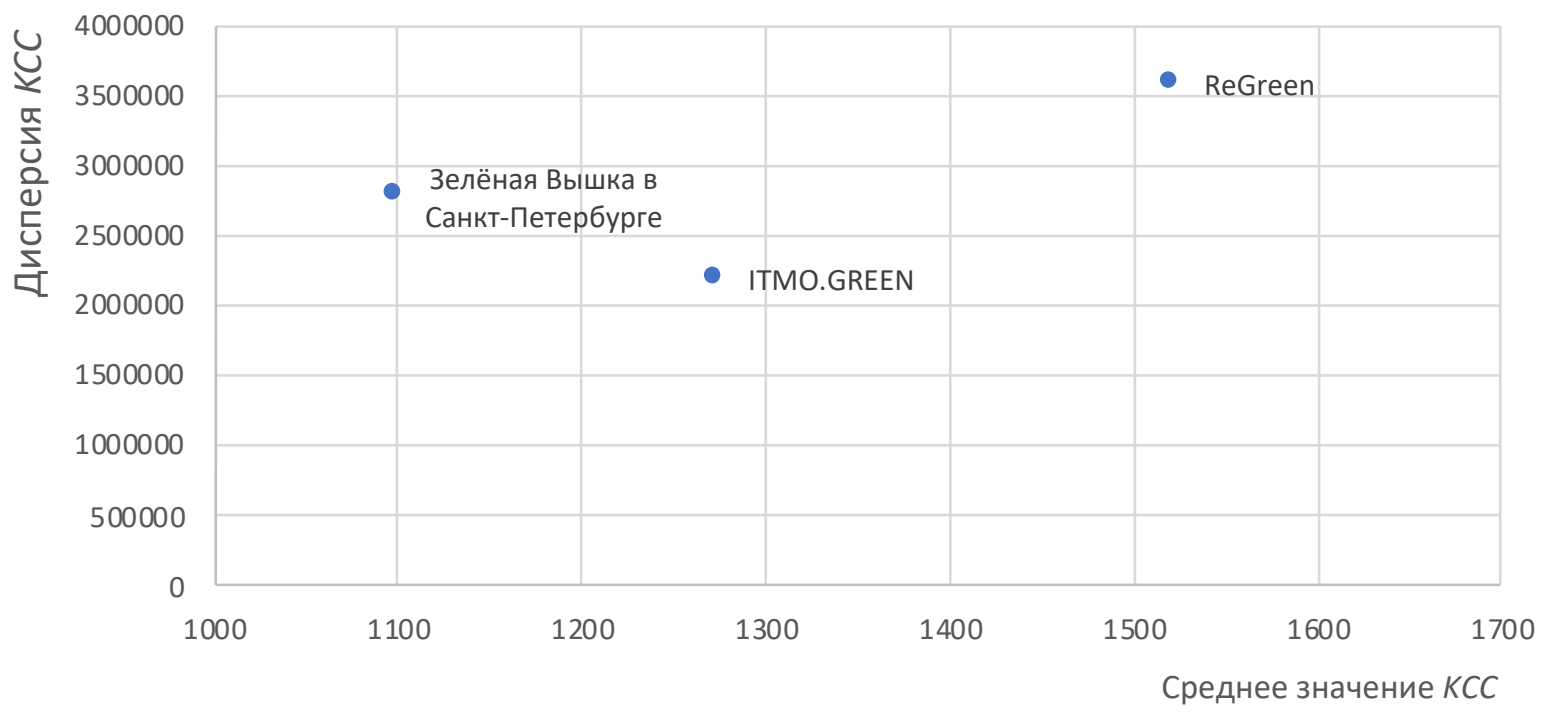

Рисунок 3. Распределение средних и дисперсий по элементам выборки 
«ITMO.GREEN», что указывает на наиболее стабильный уровень контента с точки зрения наличия в нем содержательно-тематической компоненты устойчивого потребления.

Таким образом, сформированный инструмент позволяет оценить уровень устойчивого потребления, или степень устойчивости потребления, для целей сравнительного анализа информационных субъектов или их совокупностей. На основе данного сравнительного анализа может быть определен целевой сегмент управленческого или маркетингового воздействия, а также идентифицирована совокупность потенциальных потребителей продуктов, КФУ которых является принадлежность к концепции устойчивого потребления. Следовательно, для целей дальнейшего развития данного инструмента, необходимо его последовательное применение к различным выборкам и формулирование высокоуровневых выводов.

Данный инструмент является перспективным способом анализа данных, так как он позволяет квантифицировать информационную среду с точки зрения развития концепции устойчивого потребления. В настоящее время существенную часть жизни человека занимает цифровая среда, а с началом Ковид-19 кризиса ее значимость только возросла. Возможность провести сравнительный анализ в контексте широко распространенного информационного пространства позволит оценить уровни устойчивого потребления различных субъектов относительно друг друга и в конечном итоге сформировать меры по повышению данного показателя.

Дальнейшее использование предложенного в данном исследовании инструмента может осуществляться применительно не только к тематическим экологическим объединениям, но и к официальным ресурсам различных компаний. На данный момент осуществляется повсеместное повышение уровня устойчивости в различных секторах экономики. Благодаря сравнительному анализу социальных сообществ, характеризующихся принадлежностью к определенной компании, можно выявить тенденции внедрения принципов устойчивого развития в корпоративную культуру данных компаний. По результатам исследования корпорации смогут разработать меры по повышению устойчивости и привлечению потребителей.

Кроме того, сравнительный анализ различных социальных сообществ потенциальных потребителей может показать наиболее перспективную аудиторию для распространения информации об экологически дружественной продукции с точки зрения производителей эко-товаров. Предложенный инструмент позволит повысить эффективность рекламных кампаний и тем самым повлияет на спрос на данную категорию продукции.

Данный инструмент может использоваться наиболее эффективно при комбинировании с предварительным социологическим анализом различных социальных групп потребителей с целью выявления средств воздействия на них в цифровой среде в контексте особенностей данных групп.

Для повышения уровня устойчивого потребления осуществляются такие меры как проведение экологических акций и мероприятий с полным освещением в официальных источниках компаний-организаторов и распространением информации по тематическим социальным сообществам. Кроме того, компании внедряют экологически дружественные предложения для потребителей, которые выражаются в снижении стоимости товара, и распространяют официальные подтверждения в информационных источниках о следовании принципам устойчивого развития. Поскольку ранее авторами была выдвинута гипотеза о связи осознанности и устойчивого потребления, необходимо реализовать действия, направленные именно на повышение осознанности у определенной аудитории цифрового пространства.

Таким образом, в данной работе авторами было проведено исследование концепции устойчивого потребления, предложен алгоритм сравнительного анализа уровня устойчивого потребления в контексте цифровой среды и сформированы рекомендации по использованию данного инструмента и повышению устойчивого потребления. Тематика исследования является очень актуальной на данный момент, что обусловлено тяжелой экологической обстановкой и тенденциями внедрения концепции устойчивого развития на различных уровнях экономического сектора в разных странах мира.

Цель исследования заключалась в том, чтобы построить инструмент сравнительной оценки уровня устойчивого потребления и предложить мероприятия по повышению данного показателя. Данная цель была полностью достигнута авторами в работе. 
В процессе построения аналитического инструмента были проведены первичная и вторичная токенизации при помощи инструментов компьютерной лингвистики, а также сформирован аналитический Dataframe для источника, отражающего исследуемую содержательнотематическую компоненту. Для автоматизации процесса формирования совокупности токенов был использован язык программирования Python 3.

Предложенный инструмент может быть использован для определения уровня устойчивого потребления в различных социальных сообще- ствах относительно друг друга. Данный подход может использовать компаниями как для продвижения экологически дружественной продукции, так и для привлечения потребителей посредством повышения уровня устойчивости в корпоративной культуре на основе полученных результатов исследования.

Уровень устойчивого потребления может быть повышен за счет акцентирования внимания на осознанности потребителей, выгодных предложений эко-товаров и освещения в цифровой среде экологичных акций и мероприятий.

\section{Библиографический список}

1. Gârdan D.A., Epuran G., Gârdan I.P. CREATING VALUE WITHIN CONSUMPTION CULTURE // Stud. Sci. Res. Econ. Ed. 2016. № 24.

2. Yurevich A. V. From excessive consumption to being an "excessive consumer"// Psikholog. Zh. 2020. T. 41, № 3.

3. Chukwu B.A., Kanu E. C., Ezeabogu A. N. the Impact of Advertising on Consumers Buying Behaviour // International Journal of Arts and Commerce. 2019. T. 8, № 1.

4. McInnes R.J. Sustainable development goals // The Wetland Book: I: Structure and Function, Management, and Methods. 2018.

5. Thecamp. Plastic pollution in the ocean // Xx. 2017. № March.

6. IPCC. IPCC Special Report on the Ocean and Cryosphere in a Changing Climate // Intergovernmental Panel on Climate Change. 2019.

7. Prendergast G.P., Tsang A. S.L. Explaining socially responsible consumption // J. Consum. Mark. 2019. T. 36, № 1.

8. Norwegian Ministry of the Environment. Oslo Rountable on Sustainable Production and Consumption // Nor. Minist. Environ. 1994.

9. THE 17 GOALS of Sustainable Development [Электронный ресурс]. URL: https://sdgs.un.org/goals (дата обращения: 15.11.2020).

10. Gerasimova K. Our common future // Our Common Future. 2017.

11. Sarabhai K.V. ESD for Sustainable Development Goals (SDGs) // J.Educ. Sustain. Dev. 2015. T. 9, № 2.

12. Thøgersen J. Unsustainable consumption: Basic causes and implications for policy // Eur. Psychol. 2014. T. 19, № 2.

13. Osburg V.S. и др. How detailed product information strengthens eco-friendly consumption // Manag. Decis. 2019. T. 58 , № 6 .

14. Simpson D. Institutional pressure and waste reduction: The role of investments in waste reduction resources // Int. J. Prod. Econ. 2012. T. 139, № 1.

15. Grigoryeva I.L. и др. Influence of Thermal Pollution on the Ecological Conditions in Cooling Reservoirs // Water Resour. 2019. T. 46.

16. Verain M.C.D., Dagevos H., Antonides G. Sustainable food consumption. Product choice or curtailment? // Appetite. 2015. T. 91.

17. Aydin E., Kok N., Brounen D. Energy efficiency and household behavior: the rebound effect in the residential sector // RAND J.Econ. 2017. T. 48, № 3.

18. Queiro M.E. и др. Environmental impacts of marine SO2 emissions // CONCAWE Reports. 2018.

19. Shabanova M. Separate waste collection in Russia: The level, factors and potential for citizen engagement // Mir Ross. 2019. T. 28, № 3.

20. Patlins A. Improvement of Sustainability Definition Facilitating Sustainable Development of Public Transport System // Procedia Engineering. 2017. T. 192.

21. Kudeikina I., Kaija S. The Damage Caused to the Environment in the Context of the Sustainable Development of Society // Eur. J. Sustain. Dev. 2019. T. 8, № 5.

22. Родионов Д. Г., Конников Е. А., Алферьев Д. А. Информационный капитал предприятия как целевой показатель развития в рамках цифровых экономических систем. Экономические науки. 2020. № 190. С. 131-137. 
23. Кобичева А. М., Калинина О.В., Родионов Д. Г. Университет в роли инновационной корпорации. Российский экономический интернет-журнал. 2019. № 4. С. 73.

24. Конникова О.А., Конников Е. А. Синтаксический анализ поведения потребителей в цифровой среде // В Сб.: Маркетинг сотворчества и глобальные коммуникации доверия. СПб.: Изд-во СПбГЭУ, 2020. С. $132-145$.

25. Юлдашева О. У., Соловьева Ю. Н., Ширшова О. И., Конникова О. А. Измерение устойчивого поведения потребителей. Маркетинг и маркетинговые исследования. 2019. № 3. С. 170-178. 